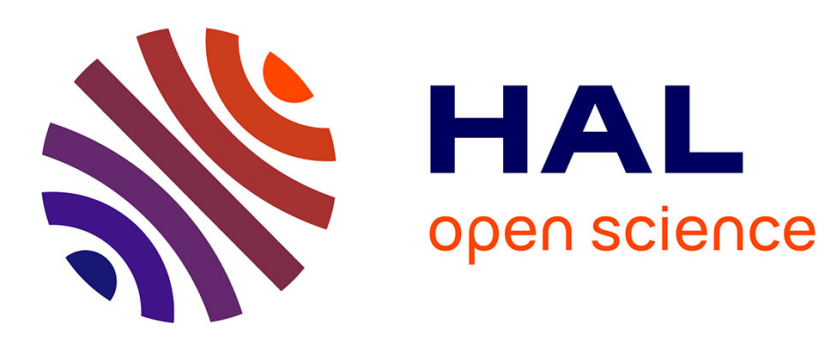

\title{
A Priori Tests of RANS Models for Turbulent Channel Flows of a Dense Gas
}

\author{
Luca Sciacovelli, Paola Cinnella, Xavier Gloerfelt
}

\section{To cite this version:}

Luca Sciacovelli, Paola Cinnella, Xavier Gloerfelt. A Priori Tests of RANS Models for Turbulent Channel Flows of a Dense Gas. Flow, Turbulence and Combustion, 2018, 101 (2), pp.295-315. 10.1007/s10494-018-9938-y . hal-02443996

\section{HAL Id: hal-02443996 https://hal.science/hal-02443996}

Submitted on 17 Jan 2020

HAL is a multi-disciplinary open access archive for the deposit and dissemination of scientific research documents, whether they are published or not. The documents may come from teaching and research institutions in France or abroad, or from public or private research centers.
L'archive ouverte pluridisciplinaire HAL, est destinée au dépôt et à la diffusion de documents scientifiques de niveau recherche, publiés ou non, émanant des établissements d'enseignement et de recherche français ou étrangers, des laboratoires publics ou privés. 


\title{
A Priori Tests of RANS Models for Turbulent Channel Flows of a Dense Gas
}

\author{
Luca Sciacovelli $^{1}$ (D) Paola Cinnella ${ }^{2}$ - Xavier Gloerfelt ${ }^{2}$
}

\begin{abstract}
Dense gas effects, encountered in many engineering applications, lead to unconventional variations of the thermodynamic and transport properties in the supersonic flow regime, which in turn are responsible for considerable modifications of turbulent flow behavior with respect to perfect gases. The most striking differences for wall-bounded turbulence are the decoupling of dynamic and thermal effects for gases with high specific heats, the liquid-like behavior of the viscosity and thermal conductivity, which tend to decrease away from the wall, and the increase of density fluctuations in the near wall region. The present work represents a first attempt of quantifying the influence of such dense gas effects on modeling assumptions employed for the closure of the Reynolds-averaged Navier-Stokes equations, with focus on the eddy viscosity and turbulent Prandtl number models. For that purpose, we use recent direct numerical simulation results for supersonic turbulent channel flows of PP11 (a heavy fluorocarbon representative of dense gases) at various bulk Mach and Reynolds numbers to carry out a priori tests of the validity of some currently-used models for the turbulent stresses and heat flux. More specifically, we examine the behavior of the modeled eddy viscosity for some low-Reynolds variants of the $k-\varepsilon$ model and compare the results with those found for a perfect gas at similar conditions. We also investigate the behavior of the turbulent Prandtl number in dense gas flow and compare the results with the predictions of two well-established turbulent Prandtl number models.
\end{abstract}

Keywords Dense gas · Compressible turbulence $\cdot$ RANS models

Luca Sciacovelli

1sciacov@caltech.edu

Paola Cinnella

paola.cinnella@ensam.eu

Xavier Gloerfelt

xavier.gloerfelt@ensam.eu

1 Mechanical and Civil Engineering Department, California Institute of Technology, Pasadena, CA, USA

2 Laboratoire DynFluid, Arts et Métiers ParisTech, Paris, France 


\section{Introduction}

Turbulent dense gas flows -i.e. flows of molecularly complex organic gasses working at temperatures and pressures of the general order of magnitude of the thermodynamic liquid/vapor critical point-, are of interest for a wide range of engineering applications. These include chemical transport and processing [1], heat pumps [2], and organic Rankine cycles $[3,4]$. The dynamics of dense gasses is governed by the fundamental derivative of gas dynamics [5] $\Gamma \equiv 1+\left.\frac{\rho}{c} \frac{\partial c}{\partial \rho}\right|_{s}$ (where $\rho$ is the density, $p$ the pressure, $s$ the entropy, and $c$ the sound speed), a measure of the rate of change of the sound speed in isentropic transformations. For dense gasses, $\Gamma<1$ for a range of thermodynamic conditions close to the saturation curve. As a consequence, the speed of sound increases in isentropic expansions and decreases in isentropic compressions, an opposite behavior with respect to the case of perfect gasses. Even larger deviations from the perfect gas behavior are expected for a class of dense gasses known as Bethe-Zel'dovich-Thompson (BZT) fluids, for which a region of $\Gamma<0$ exists. The reader is referred to [6] and references cited therein for more information about dense gas dynamics. The peculiar thermodynamic behavior of dense gasses requires using advanced equations of state in place of the simple perfect gas law. Moreover, in the dense gas regime, the dynamic viscosity $\mu$ and the thermal conductivity $\lambda$ exhibit a significant dipendency on both temperature and pressure, instead of temperature only. Similarly, the approximation of nearly constant molecular Prandtl number is no longer valid. Numerical simulations of turbulent dense gas flows of engineering interest are based on the Reynolds-Averaged Navier-Stokes (RANS) equations, which need to be supplemented by a model for the Reynolds stress tensor and turbulent heat flux. The accuracy of RANS models for dense-gas flows has not been properly assessed up to date, due to the lack of both experimental and numerical reference data. DNS databases are then needed to quantify the deficiencies of existing turbulence models and to develop and calibrate improved ones. First contributions to the DNS of decaying homogeneous isotropic turbulence were reported in $[7,8]$, while the influence of dense gas effects in wall-bounded turbulent flows was investigated in [9].

Past attempts to assess RANS modeling approximations against direct numerical simulation (DNS) are restricted to perfect gas flows and are relatively recent, due to the need for sufficiently high-Reynolds number DNS. Huang et al. [10] use DNS results for compressible turbulent channel flows at bulk Mach numbers of 1.5 and 3 and bulk Reynolds numbers of 3000 and 4880, respectively, to discuss deviations between Reynolds and Favre averages of various flow properties and found that differences are rather small, except in the near-wall region, where moderate deviations are observed. The authors also investigate the crosswise distribution of the exact turbulent Prandtl and show that it is globally similar to the one found in incompressible flow, with a plateau at about $y^{*} \approx 50$ (see Eq. 6 for a definition), i.e. farther away from the wall than in the incompressible case. Patel et al. [11] review several near-wall and low-Reynolds turbulence models for incompressible flows. Experimental data in the near wall region are limited and suffer from probe interference effects and the determination of the wall shear-stress. They show that experimental data for the average velocities, turbulent kinetic energy $k$, shear stress, eddy viscosity $v_{t}$ and dissipation $\varepsilon$ are in good agreement with the asymptotic behavior predicted by Launder [12] by expanding the exact incompressible equations. On the other hand, they consider seven variants of the $k-\varepsilon$ model and the Wilcox-Rubesin $k-\omega$ model. For the former class of models, $v_{t}=C_{\mu} f_{\mu} k^{2} / \widetilde{\varepsilon}$, where usually $C_{\mu}=0.09, f_{\mu}$ is a near-wall damping function and $\widetilde{\varepsilon}$ is a near wall dissipation, while for $k-\omega$ one has $v_{t}=f_{\mu} k / \omega$. A posteriori 
tests of the models for a set of turbulent boundary layer flows show that the scatter in the results is very high and that no model is clearly better than the other ones for all cases. The authors conclude that most modifications to high-Reynolds models lack a physically sound basis. All models are highly sensitive to the closure coefficients. The damping function $f_{\mu}$ is also found to have a predominant influence on the model performance compared to other damping functions and low-Reynolds extra terms in the transport equations of the turbulent variables. Similar remarks can be found in the work of Shih [13], who presents an improved $k-\varepsilon$ model for the near-wall region and carries out a posteriori tests against DNS data for an incompressible turbulent channel flow (TCF) [14]. The author observes that most lowReynolds $k-\varepsilon$ models do not verify the theoretical asymptotic behavior near the wall. He also finds that the agreement with the DNS data tends to improve when the Reynolds number is increased. Durbin [15] investigates near-wall turbulence models providing the correct asymptotic behavior without need for damping functions. A priori tests of $v_{t}$ values obtained by using DNS data and the classical eddy viscosity formulation based on $k$ and $\varepsilon$ show large discrepancies with respect to the "exact" eddy viscosity (estimated from DNS data for TCF as the ratio of the turbulent shear stress to the wall normal derivative of the streamwise velocity) for any choice of the constant $C_{\mu}$. To recover the correct trend, Durbin suggests the use of an alternative energy scale based on the wall-normal fluctuating speed, leading to $v_{t}=C_{\mu} \overline{v^{\prime 2}} k / \varepsilon$. Although such a choice does not ensure the theoretical $y^{+3}$ trend near the wall ( $v_{t} \sim y^{+4}$ instead), it accounts effectively for the blocking effect of the wall, which has a major influence on near-wall behavior. A priori tests of eddy viscosity models against incompressible TCF data (and specifically the high-Reynolds DNS of [16]) are carried out by Karimpour and Venayagamoorthy [17], where Durbin's formulation is revisited by introducing a length scale based on the dissipation and the mean shear for the near wall region. The models under investigation are found to agree better and better with DNS data as the Reynolds number is increased. He et al. [18] investigate the validity of RANS models for supercritical fluid flows, characterized by strong variations of the fluid properties both with temperature and pressure (a feature also found in dense gases) that may affect turbulence production, loss and heat transfer mechanisms. A posteriori tests of various popular lowReynolds RANS models against DNS data for supercritical fluid flows of carbon dioxide in ducts show a large scatter of the results. Among the considered models, Durbin's v2f model is found to produce the best predictions. On the other hand, none of the models is found to provide satisfactory predictions of the heat transfer for strongly buoyant cases. This is attributed to the use of a constant turbulent Prandtl number for modeling the turbulent heat flux. Pecnik and Patel [19] investigate the effect of variable mean properties on turbulence modeling. More specifically, they reformulated the kinetic energy equation for a variableproperty fluid and showed that semi-local scaling can be used to recast the turbulent kinetic energy equation into the classical form for incompressible flows, provided the production term is rewritten in terms of the van Driest velocity and the dissipation tensor rescaled with the semi-local friction Reynolds number. Their a posteriori tests (using DNS data to rule out errors coming from the closure terms in the energy equation) indicate that solving RANS equations in the semi-locally scaled form improves the agreement with reference DNS data. Finally, Irrenfried and Steiner [20] review the P-function approach for heat-transfer modeling in the case of (constant) high Prandtl-number incompressible flows. The role of the eddy viscosity model and of the Prandtl number model are investigated by means of comparisons with DNS data for turbulent pipe flows at Prandtl numbers ranging from 1 to 20.

In this work, we use recent DNS results [9] for supersonic turbulent channel flows of PP11 (a heavy fluorocarbon representative of dense gases) to carry out a priori tests of the 
validity of some currently-used models for the turbulent stresses and heat flux. Specifically, we examine the behavior of the modeled eddy viscosity for some low-Reynolds variants of the $k-\varepsilon$ model for dense gas flows at various bulk Mach and Reynolds numbers and compare the results with those found in perfect gas TCF at similar conditions. It should be noted that many eddy viscosity models sacrifice the accurate representation of transported turbulent quantities such as turbulent kinetic energy or dissipation in favor of a better match of the computed eddy viscosity with reference (mostly experimental) data. Indeed, some models are specifically tuned for "unphysical" values of the turbulent properties, although they may lead to reasonably accurate a posteriori results for many classes of flows. In this respect, a priori tests are useful to check the physical soundness of such models and to evaluate their ability to reproduce exact trends derived from DNS data more than to assess their predictive accuracy. We also investigate the behavior of the turbulent Prandtl number in dense gas flow and compare the results with the predictions of two well-established turbulent Prandtl number models. For this purpose, in Section 2 we briefly recall the numerical models and methods used to generate the DNS database, then in Section 3 we provide an overview of the most relevant DNS results with focus on dense-gas effects. Finally, in Section 4 we examine the validity of some low-Reynolds eddy viscosity models and turbulent Prandtl number models and show how the results are affected by the peculiar thermodynamic and transport-property variations typical of dense gas flows.

\section{Governing Equations and Numerical Method}

Dense gas flows are governed by the single-phase compressible Navier-Stokes equations. For the present channel flow calculations, a source term $f_{u_{i}}$ is added to the streamwise momentum equation to counteract wall friction and maintain a target bulk mass flow [21]. A corresponding term is introduced in the energy equation.

The governing equations are supplemented by thermal and caloric equations of state, respectively:

$$
p=p(\rho, T) \quad \text { and } \quad e=e(\rho, T),
$$

related by means of the compatibility condition:

$$
e=e_{r}+\int_{T_{r}}^{T} c_{v, \infty}\left(T^{\prime}\right) \mathrm{d} T^{\prime}-\int_{\rho_{r}}^{\rho}\left[\left.T \frac{\partial p}{\partial T}\right|_{\rho}-p\right] \frac{\mathrm{d} \rho^{\prime}}{\rho^{\prime 2}},
$$

where the subscript $(\bullet)_{r}$ indicates a reference state, $c_{v, \infty}(T)$ is the specific heat at constant volume in the ideal-gas limit, and the superscript $(\bullet)^{\prime}$ denotes auxiliary integration variables.

The present calculations are based on the Martin-Hou (MAH) thermal equation of state [22], which is reasonably accurate for the fluid of interest and requires a minimum amount of experimental information for setting the gas-dependent coefficients. The MAH equation involves five virial terms and reads:

$$
p=\frac{R T}{(v-b)}+\sum_{i=2}^{5} \frac{f_{i}(T)}{(v-b)^{i}},
$$

with $b=v_{c r}\left[1-\left(20.533-31.883 Z_{c r}\right) / 15\right]$ and $f_{i}(T)=A_{i}+B_{i} T+C_{i} \exp \left(-k T / T_{c r}\right)$, where $v=1 / \rho$ denotes the specific volume, $v_{c r}, T_{c r}$ and $Z_{c r}$ are the critical specific volume, temperature and compressibility factor, and $k=5.475$. The coefficients $A_{i}, B_{i}$ and $C_{i}$ depend on the critical temperature and pressure, the critical compressibility factor, the 
Boyle temperature and one point on the vapor pressure curve. Variations of the low-density specific heat with temperature are modeled by means of a power law:

$$
c_{v_{\infty}}(T)=c_{v_{\infty}}\left(T_{c r}\right)\left(\frac{T}{T_{c r}}\right)^{n}
$$

where $n$ is a material-dependent parameter. In addition to the equations of state, suitable models for the dynamic viscosity $\mu$ and thermal conductivity $\lambda$ of dense gases, which depend on both the gas temperature and pressure, have to be specified. The present results are based on the model of Chung et al. [23], which incorporates a correction term in the dense-gas region. Such a model has been extensively used in previous works on dense gases (e.g. $[24,25])$ and is considered to be a reasonably accurate semi-theoretical model for calculating the viscosity based on the knowledge of a few thermophysical input parameters (see [26] for more details). A full description of the model equations is given in [9], Appendix A.

The dense fluid considered in the following simulations is the perfluoro-perhydro phenanthrene, (chemical formula $\mathrm{C}_{14} \mathrm{~F}_{24}$ ), called hereafter with its commercial name PP11. Its main thermodynamic properties, extracted from [27], are provided in Table 1. DNS results are also presented for air, modeled as a polytropic perfect gas. In the latter case, the viscosity is assumed to follow a power law of the temperature and the thermal conductivity is computed according to a constant Prandtl number assumption $(\mathrm{Pr}=0.7)$.

The simulations are carried out by means of an in-house CFD code [28] based on high-order dispersion-relation preserving finite difference schemes. The derivatives of the convective fluxes are approximated by using a fourth-order optimized scheme on an elevenpoint stencil [29]. Standard fourth-order finite differences are used for the discretization of the viscous fluxes. Non-uniform mesh sizes are taken into account by means of coordinate transforms. The mesh stretching rate in the wall-normal direction is kept below $1.5 \%$ to ensure accuracy. An optimized selective sixth-order filter [30] is applied in each direction to eliminate grid-to-grid oscillations. Time integration is based on a low-storage optimized six-step Runge-Kutta scheme [29].

\section{Summary of DNS Results}

In [9] the TCF configuration was simulated for a dense and a perfect gas at various Mach and Reynolds numbers. The computational domain (sketched in Fig. 1) has dimensions $L_{x} \times$ $L_{y} \times L_{z}=8 \pi h \times 2 h \times 2 \pi h$ for all cases. Periodic conditions are applied in the streamwise $(x)$ and spanwise $(z)$ directions, and isothermal no-slip wall conditions are applied at the lower and upper walls.

Table 1 Thermodynamic properties of PP11 $\left(\mathrm{C}_{14} \mathrm{~F}_{24}\right)$ : molecular weight $(\mathcal{M})$, critical temperature $\left(T_{c r}\right)$, critical density $\left(\rho_{c r}\right)$, critical pressure $\left(p_{c r}\right)$, critical compressibility factor $\left(Z_{c r}\right)$, acentric factor $\left(\omega_{a c}\right)$, dipole moment of the gas phase $(\xi)$, boiling temperature $\left(T_{b}\right)$, ratio of ideal-gas specific heat at constant volume over the gas constant $\left(c_{v}\left(T_{c r}\right) / R\right)$ at the critical point, and parameter for the low-density specific heat power law $(n)$

\begin{tabular}{llllllllll}
\hline $\mathcal{M}$ & $\mathrm{T}_{c r}$ & $\rho_{c r}$ & $p_{c r}$ & $Z_{c r}$ & $\omega_{a c}$ & $\xi$ & $T_{b}$ & $c_{v}\left(T_{c r}\right) / R$ & $n$ \\
$\mathrm{~g} \mathrm{~mole}^{1}$ & $\mathrm{~K}$ & $\mathrm{~kg} \mathrm{~m}^{3}$ & $\mathrm{MPa}$ & - & - & $\mathrm{D}$ & $\mathrm{K}$ & - & - \\
624.11 & 650.2 & 627.14 & 1.46 & 0.2688 & 0.4963 & 0.0 & 488.15 & 97.3 & 0.5776 \\
\hline
\end{tabular}




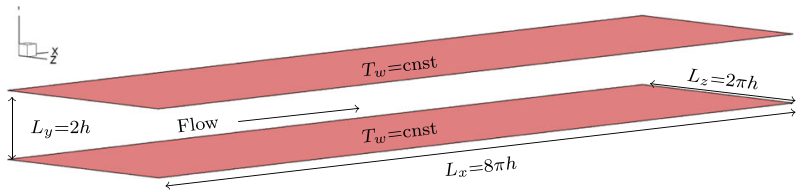

Fig. 1 Sketch of the computational domain

In the following, the subscripts $(\bullet)_{B},(\bullet)_{w}$ and $(\bullet)_{c l}$ denote averaging over the whole computational domain, the walls and centerline, respectively; $\overline{(\bullet)}$ indicates Reynolds averaging over the homogeneous spatial directions and in time, whereas $(\bullet)^{\prime}$ are Reynolds fluctuations; similarly, $\widetilde{(\bullet)}$ and $(\bullet)^{\prime \prime}$ denote Favre averaging and Favre fluctuations. In the following, we also use the centerline Reynolds and Mach numbers, respectively defined as $R e_{c l}=\bar{\rho}_{c l} \bar{u}_{c l} h / \bar{\mu}_{c l}$ and $M_{c l}=\bar{u}_{c l} / \bar{c}_{c l}$. The DNS conditions are defined by setting the bulk Reynolds number $\operatorname{Re}_{B}$ and the bulk Mach number $M_{B}$, defined as:

$$
R e_{B}=\frac{\rho_{B} \tilde{u}_{B} h}{\bar{\mu}_{w}}, \quad M_{B}=\frac{\tilde{u}_{B}}{\bar{c}_{w}},
$$

where $\rho_{B}$ is the bulk density, $h$ is the channel half-height, $\bar{\mu}_{w}$ and $\bar{c}_{w}$ are the dynamic viscosity and the sound speed at the walls, respectively. In the definition of the bulk reference numbers, wall values for viscosity and speed of sound are usually considered since they can be easily controlled through the wall temperature in the case of a perfect gas. Unfortunately, this is no longer possible when using more complex thermodynamic models, since the speed of sound and the transport properties depend on both temperature and density values and $\bar{\rho}_{w}$ is not known a priori. In these cases, the conditions are adjusted iteratively by starting with a reasonable guess for the ratio $\bar{\rho}_{w} / \rho_{B}$. Once this preliminary calculation is converged, the $\bar{\rho}_{w}$ obtained from the simulation is used to compute the updated values of $\bar{\mu}_{w}$ and $\bar{c}_{w}$, and the flow field is interpolated onto a new grid (adapted to the updated value of the Reynolds number). In order to achieve convergence to the desired state, about 3 to 4 iterative cycles are needed.

The main numerical parameters of the DNS databases are reported in Table 2, where each case is assigned a tag of the form $\mathrm{XM} \alpha \mathrm{R} \beta$. The first letter indicates the fluid (A for air and P for PP11), $\alpha$ refers to $M_{B}\left(\alpha=1,2,3\right.$ for $M_{B}=1.5,2.25,3$, respectively) and $\beta$ to $\operatorname{Re}_{B}\left(\beta=3,7,12\right.$ for $\operatorname{Re}_{B}=3000,7000,12000$, respectively). A smaller set of cases with respect to that presented in [9] is retained here for the purpose of clarity. This subset allows indeed to study the influence of a fixed $M_{B}$ with varying $R e_{B}$, and vice-versa.

DNS results are presented in semi-local scaling [10], based on both wall and centerline quantities

$$
y^{*}:=\frac{\bar{\rho}(y) u_{\tau}^{*}\left(y-y_{w}\right)}{\bar{\mu}(y)} ; \quad \operatorname{Re}_{\tau, c l}^{*}:=\frac{\bar{\rho}_{c l} u_{\tau}^{*} h}{\bar{\mu}_{c l}}=y^{*}(y=h)
$$

(being $u_{\tau}^{*}=\sqrt{\bar{\tau}_{w} / \bar{\rho}(y)}$ the semi-local friction velocity and $\bar{\tau}_{w}=\overline{\left(\mu \frac{\partial u}{\partial y}\right)_{w}}$ the shear stress at the wall), which has proven to give quite satisfactory results in collapsing first- and second-order moments [31-33] obtained from a wide range of $M_{B}$ and $R e_{B}$.

The computational grids are chosen accordingly in order to provide a good spatial resolution in all directions. Specifically, the chosen spatial resolutions, expressed in semi-local units, are in the ranges $\Delta x^{*} \in[10,16], \Delta y_{w}^{*} \in[0.5,0.8]$, and $\Delta z^{*} \in[4,6]$. The spatial resolution is also evaluated with respect to the wall-normal distribution of the local 
Table 2 Numerical parameters and DNS results for air and PP11 cases

\begin{tabular}{|c|c|c|c|c|c|c|c|c|c|c|}
\hline \multirow[b]{2}{*}{ Case } & \multicolumn{5}{|l|}{ Air } & \multicolumn{5}{|c|}{ PP11 } \\
\hline & AM1R3 & AM1R7 & AM2R7 & AM3R7 & AM3R12 & PM1R3 & PM1R7 & PM2R7 & PM3R7 & PM3R12 \\
\hline$M_{B}$ & 1.5 & 1.5 & 2.25 & 3.0 & 3.0 & 1.5 & 1.5 & 2.25 & 3.0 & 3.0 \\
\hline$R e_{B}$ & 3000 & 7000 & 7000 & 7000 & 12000 & 3000 & 7000 & 7000 & 7000 & 12000 \\
\hline$N_{x}$ & 512 & 768 & 768 & 1024 & 1536 & 512 & 768 & 768 & 768 & 1280 \\
\hline$N_{y}$ & 256 & 384 & 384 & 512 & 768 & 256 & 384 & 384 & 384 & 768 \\
\hline$N_{z}$ & 256 & 512 & 512 & 768 & 1024 & 256 & 512 & 512 & 512 & 1024 \\
\hline$R e_{\tau_{w}}$ & 218.7 & 466.5 & 538.6 & 627 & 1017 & 191.3 & 401.6 & 404.6 & 425.1 & 692.8 \\
\hline$R e_{\tau, c l}^{*}$ & 147.1 & 314.8 & 252.0 & 199.7 & 324.7 & 196.4 & 412.1 & 428.0 & 492.1 & 800.2 \\
\hline$M_{c l}$ & 1.5 & 1.47 & 1.9 & 2.18 & 2.16 & 1.62 & 1.60 & 2.25 & 2.61 & 2.58 \\
\hline$M_{\tau_{w}}$ & 0.08 & 0.07 & 0.09 & 0.11 & 0.1 & 0.09 & 0.08 & 0.12 & 0.15 & 0.14 \\
\hline$R e_{c l}$ & 2740 & 6319 & 5071 & 4035 & 6905 & 3659 & 8423 & 8866 & 10256 & 17618 \\
\hline$R e_{B b}$ & 2417 & 5627 & 4582 & 3690 & 6416 & 3148 & 7346 & 7728 & 8944 & 15498 \\
\hline$-B_{q} \times 10^{3}$ & 48.8 & 44.6 & 87.0 & 133 & 126 & 0.26 & 0.24 & 0.44 & 0.58 & 0.56 \\
\hline $\bar{\rho}_{w} / \rho_{B}$ & 1.36 & 1.37 & 1.84 & 2.49 & 2.51 & 1.05 & 1.05 & 1.11 & 1.25 & 1.25 \\
\hline $\bar{\rho}_{c l} / \rho_{B}$ & 0.98 & 0.99 & 0.97 & 0.96 & 0.97 & 1.00 & 1.00 & 1.00 & 0.99 & 0.99 \\
\hline $\bar{T}_{c l} / T_{w}$ & 1.39 & 1.39 & 1.88 & 2.59 & 2.58 & 1.00 & 1.00 & 1.00 & 1.01 & 1.01 \\
\hline $\bar{\mu}_{c l} / \bar{\mu}_{w}$ & 1.26 & 1.26 & 1.56 & 1.95 & 1.94 & 0.95 & 0.95 & 0.90 & 0.77 & 0.77 \\
\hline $\bar{c}_{c l} / \bar{c}_{w}$ & 1.18 & 1.18 & 1.37 & 1.61 & 1.61 & 1.07 & 1.07 & 1.14 & 1.32 & 1.32 \\
\hline $\operatorname{Pr}_{B}$ & 0.7 & 0.7 & 0.7 & 0.7 & 0.7 & 2.39 & 2.39 & 2.36 & 2.31 & 2.31 \\
\hline
\end{tabular}

$M_{B}$ and $\operatorname{Re}_{B}$ are the bulk Mach and Reynolds numbers; $N_{x}, N_{y}$ and $N_{z}$ are the numbers of grid points in the streamwise, wall-normal and spanwise directions; $R e_{\tau_{w}}$ is the friction Reynolds number; $R e_{\tau, c l}^{*}$ is the centerline semi-local friction Reynolds number; $M_{c l}=\bar{u}_{c l} / \bar{c}_{c l}$ is the centerline Mach number; $\operatorname{Re}_{c l}=$ $\bar{\rho}_{c l} \bar{u}_{c l} h / \bar{\mu}_{c l}$ the centerline Reynolds number; $R e_{B b}=\rho_{B} \tilde{u}_{B} h / \mu_{B}$ the Reynolds number based on bulk conditions; $B_{q}=\bar{q}_{w} /\left(\bar{\rho}_{w} u_{\tau} \bar{h}_{w}\right)$ the heat flux coefficient at the walls, with $\bar{h}_{w}$ the averaged specific enthalpy at the wall; $\bar{\rho}_{w} / \rho_{B}$ and $\bar{\rho}_{c l} / \rho_{B}$ the wall and centerline normalized density values; $\bar{T}_{c l} / \bar{T}_{w}, \bar{\mu}_{c l} / \bar{\mu}_{w}$ and $\bar{c}_{c l} / \bar{c}_{w}$ the normalized centerline temperature, viscosity and speed of sound; and $\operatorname{Pr}_{B}=\mu_{B} c_{p, B} / \lambda_{B}$ the bulk Prandtl number

Kolmogorov scale $\bar{\eta}_{K}=\left[(\bar{\mu} / \bar{\rho})^{3} / \bar{\varepsilon}\right]^{1 / 4}$ for the dynamic turbulent structures, and the local Batchelor scale $\bar{\eta}_{\theta}=\bar{\eta}_{K} / \overline{\operatorname{Pr}}^{1 / 2}$ for the thermal structures. The definitions of the two length-scales show that they depend on the turbulent kinetic energy dissipation $\bar{\varepsilon}$, and the thermal scales become smaller than the dynamic ones for Prandtl numbers higher than unity. According to [34] and [35], resolution requirements for well-resolved DNSs are $\Delta x<12 \eta_{K}, \Delta y<2 \eta_{K}$ and $\Delta z<6 \eta_{K}$. Figures 2 and 3 show the streamwise and wallnormal grid sizes for the different simulations in terms of $\eta_{K}$ and $\eta_{\theta}$, respectively (the line legend associated with each $\left(M_{B}, R e_{B}\right)$ couple is given in Table 3$)$. Very good resolutions are obtained in terms of dynamic scales with the selected grids, in particular, for the dense gas realizations where $\Delta x / \eta_{K}<10$ and $\Delta y / \eta_{K}<1.5$ throughout the channel. By analyzing the Batchelor scale, thermal requirements are seen to be less stringent for air (for which $\operatorname{Pr}=0.7=$ const .) and more severe for high- $M_{B}$ PP11 cases, due to the high molecular Prandtl number values that are reached at the wall (as shown later). Nevertheless, except for a few cells close to the walls for the $M_{B}=3$ cases, the profiles of $\Delta x / \eta_{\theta}$ are within DNS requirements. Additionally, the wall-normal and spanwise (not shown for brevity) grid sizes ensure an excellent thermal resolution. 

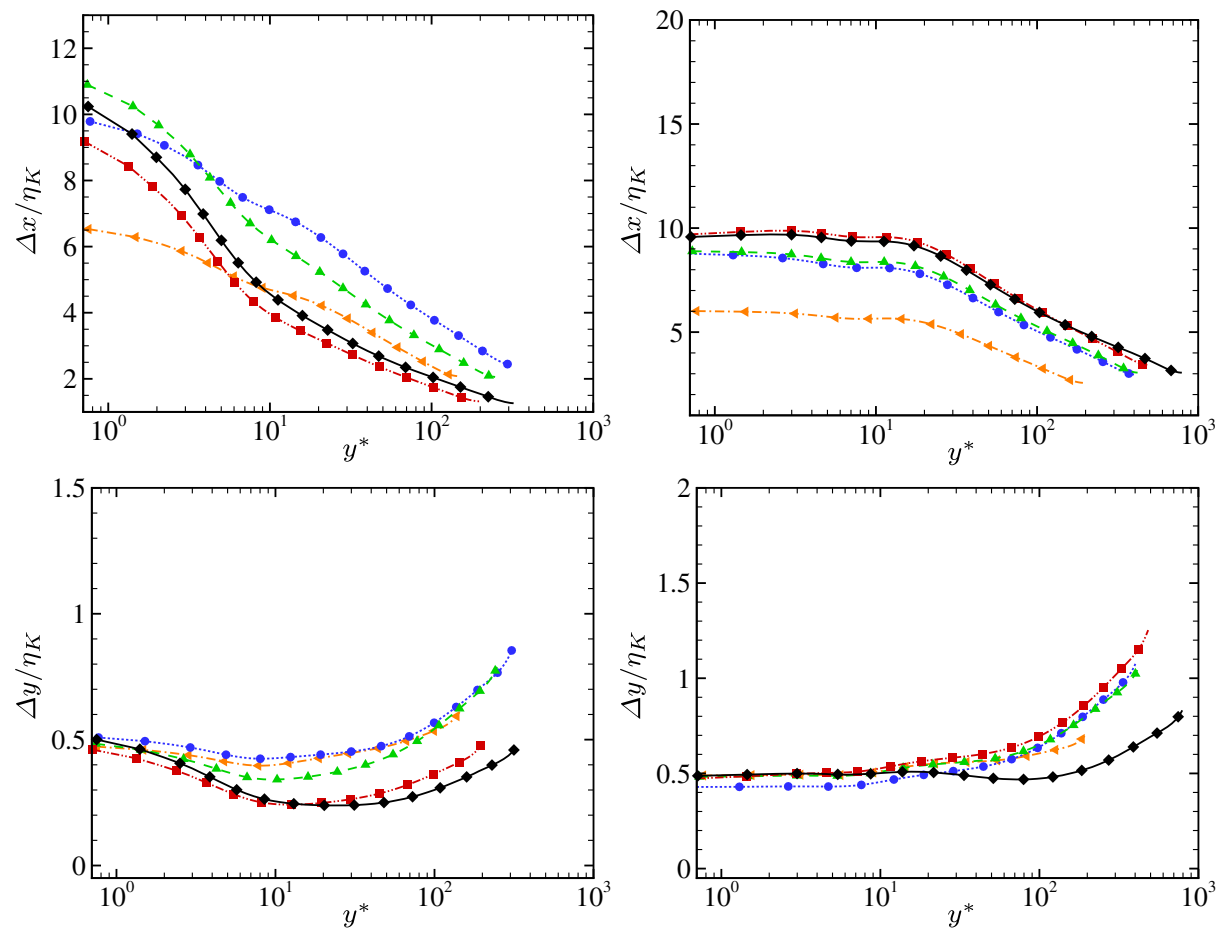

Fig. 2 Streamwise and wall-normal grid size normalized with respect to the local Kolmogorov scale $\eta_{K}$ as a function of $y^{*}$ for DNS of air (left) PP11 (right) TCF at various $M_{B}$ and $R e_{B}$. Line legend as in Table 3

The global DNS results reported in Table 2 illustrate the different behavior of the main thermodynamic and transport properties between air and PP11. For PP11, due to the large specific heat of the fluid, the average temperature is almost constant across the channel for any choice of the Mach and Reynolds numbers, and the centerline temperature differs by less than $1 \%$ of $\widetilde{T}_{w}$. Decoupling of dynamic and thermal effects in the dense gas also leads to smaller mean density variations across the channel. For air, the centerline density is up to $60 \%$ lower than $\bar{\rho}_{w}$ (at $M_{B}=3$ ) whereas variations below $20 \%$ are observed for PP11, as shown by $\overline{\rho_{w}} / \rho_{B}$ and $\overline{\rho_{c l}} / \rho_{B}$ values in Table 2 . The normalized viscosity $\bar{\mu} / \bar{\mu}_{w}$, which essentially follows temperature variations for air, varies instead like the density for PP11 and tends to decrease toward the channel center. As a consequence, the semi-local friction Reynolds number $R e_{\tau}^{*}$ increases toward the channel centerline in PP11, as shown in Fig. 4. Specifically, the dense gas flow exhibits lower values of $R e_{\tau}^{*}$ near the wall, compared to a perfect gas flow at the same bulk conditions, whereas the centerline value of $R e_{\tau}^{*}$ is much higher in the dense gas, due to the negligible friction heating in the outer region (Fig. 4). On the other hand, the average Prandtl number $\overline{\mathrm{Pr}}$, which is approximately equal to 2.3 in the outer region for all PP11 cases (against 0.7 for air), tends to increase even further near the wall (with values as high as $\sim 3.9$ for the highest Mach number), following essentially the trend of the average specific heat (see Fig. 5). Dense gas effects are stronger at higher $M_{B}$, since the local thermodynamic states spread over a wider range.

The velocity profiles (not reported for brevity) can be collapsed onto the incompressible one by using a scaling accounting for the mean flow property variations [36, 37]. 

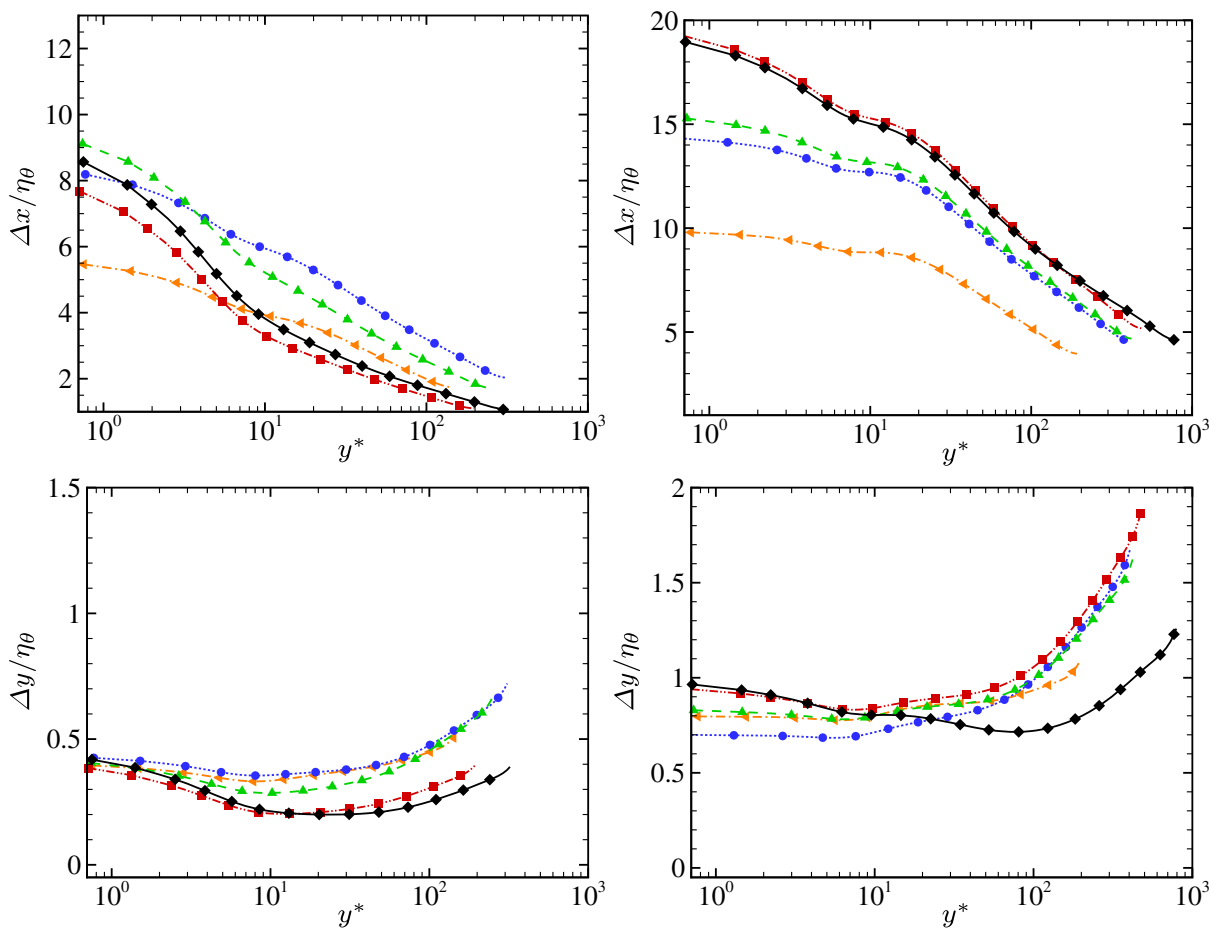

Fig. 3 Streamwise and wall-normal grid size normalized with respect to the local Batchelor scale $\eta_{\theta}$ as a function of $y^{*}$ for DNS of air (left) PP11 (right) TCF at various $M_{B}$ and $R e_{B}$. Line legend as in Table 3

Table 3 Flow cases and associated line legend

\begin{tabular}{|c|c|c|c|c|c|}
\hline$M_{B}$ & 1.5 & 1.5 & 2.25 & 3 & 3 \\
\hline $\operatorname{Re}_{B}$ & 3000 & 7000 & 7000 & 7000 & 12000 \\
\hline Line & $=-4=$ & $\cdots$ & $--\Delta-$ & $-\cdots+-\cdots$ & $\sim$ \\
\hline
\end{tabular}
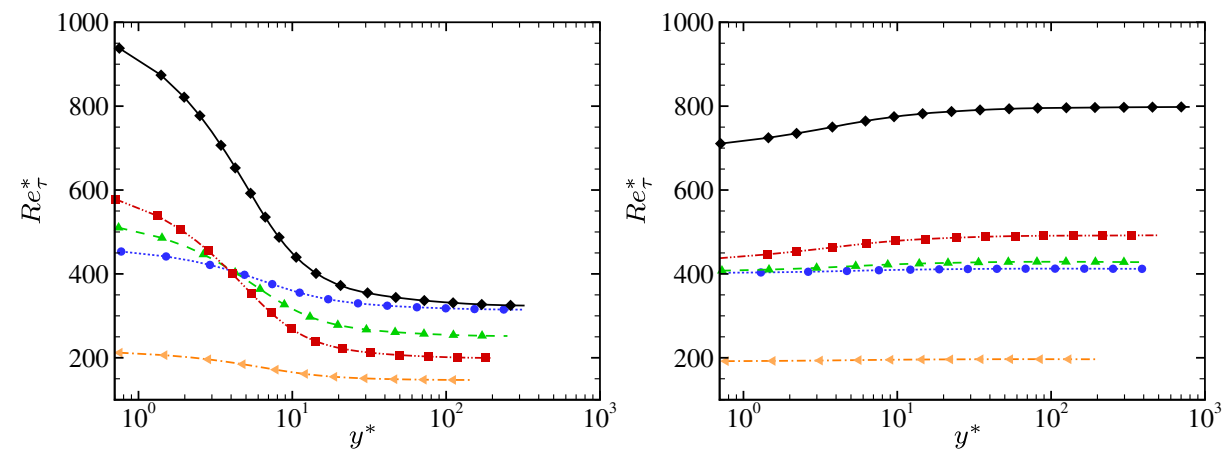

Fig. 4 Local friction Reynolds number $R e_{\tau}^{*}$ as a function of $y^{*}$ for DNS of air (left) and PP11 (right) TCF at various $M_{B}$ and $R e_{B}$. Line legend as in Table 3 

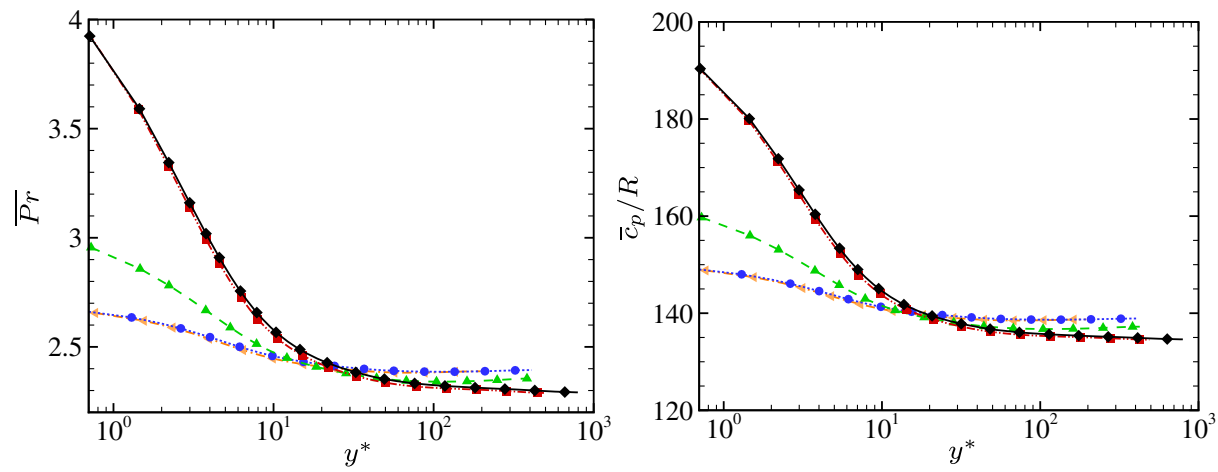

Fig. 5 From left to right: average Prandtl number $\overline{P r}$, and average isobaric specific heat normalized with the gas constant $\bar{c}_{p} / R$ as a function of $y^{*}$ for DNS of PP11 at various $M_{B}$ and $R e_{B}$. Line legend as in Table 3

For PP11, the classical van Driest velocity scaling (developed for adiabatic boundary layers) is also found to collapse the profiles rather accurately, due to the drastically reduced wall heat transfer, compared to the perfect gas. Sample results for second-order statistics are reported in Fig. 6, which displays the r.m.s. density values, the Reynolds shear stress $\overline{\rho u^{\prime \prime} v^{\prime \prime}}+=\tau_{w}^{-1} \overline{\rho u^{\prime \prime} v^{\prime \prime}}$, as well as the normalized turbulent heat flux $-\overline{\rho v^{\prime \prime} h^{\prime \prime}} q_{w}^{-1}$ (with $h^{\prime \prime}$ the Favre enthalpy fluctuation). The relative density and pressure fluctuations are of the same order of those observed for air flows (see [9]), whereas temperature fluctuations (not reported) are nearly two orders of magnitude lower. Remarkably, $\overline{\rho^{\prime 2}}$ decreases monotonically from wall to centerline, contrary to light gases. This is due to the peculiar thermodynamic behavior of PP11 at the considered conditions, as demonstrated in [9] by using the equation of state. In all cases, density fluctuations remain small compared to the mean value, and Morkovin's hypothesis is satisfied even at the highest Mach number. Despite the striking differences in the thermodynamic behavior, Reynolds stress profiles are similar to those observed, e.g., in [36] for low-Mach TCF with temperature-dependent transport properties. The liquid-like behavior of viscosity leads to an increase of the spanwise, wall-normal and Reynolds shear stresses with respect to the corresponding incompressible evolution, whereas the streamwise one decreases. This effect is stronger when increasing Mach number. Due to the small temperature gradients for PP11, the heat flux at the walls is strongly reduced with respect to air (values of the wall heat transfer coefficient $B_{q}$ are reported in Table 2); similar considerations hold for the wall-normal turbulent heat flux, which is orders of magnitude smaller in PP11 due to the reduced enthalpy fluctuations. However, when normalized with $q_{w}$, the turbulent heat flux shows similar profiles between air and dense gas cases (Fig. 6e and f): namely, $-\overline{\rho v^{\prime \prime} h^{\prime \prime}} q_{w}^{-1}$ peaks at $y^{*} \sim 12 \div 18$ for both fluids, and the peak increases with $R e_{\tau, c l}^{*}$. The higher values of $R e_{\tau, c l}^{*}$ encountered in PP11 flows result in higher turbulent heat flux to wall heat flux ratios than in the perfect gas. Figure 7 shows the ratio of production to dissipation term $P / \varepsilon$ of the turbulent kinetic energy budget. The production peak is located as usual at $y^{*} \approx 12$. For the higher $R e_{\tau, c l}^{*}$ cases, a second production peak is observed in the outer region, like in high- $R e$ incompressible flow, due to the reduced dissipation close to centerline. Lastly, a visualization of flow structures for PP11 at $M_{B}=3, R e_{B}=12000$ is provided in Fig. 8. The structures are identified by means of the $Q$-criterion, $Q=\frac{1}{2}\left(\Omega_{i j}^{2}-S_{i j}^{2}\right)$, where $\Omega_{i j}$ and $S_{i j}$ denote the rotation-rate and strain-rate tensors, respectively. 


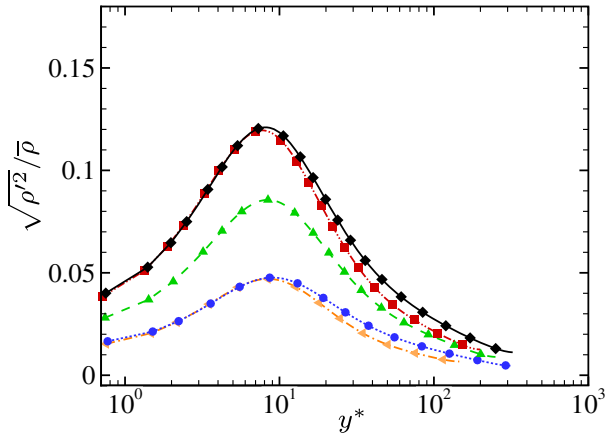

(a)

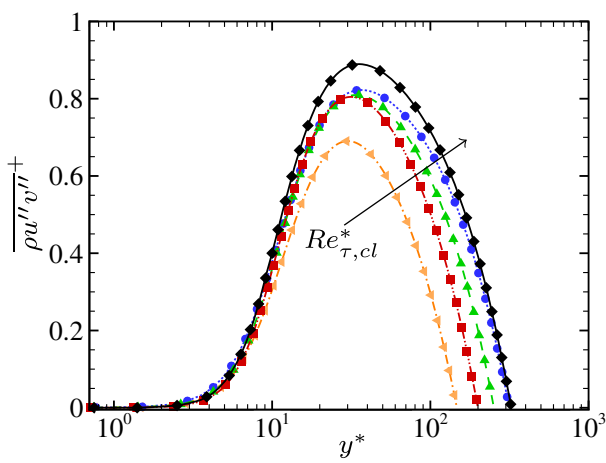

(c)

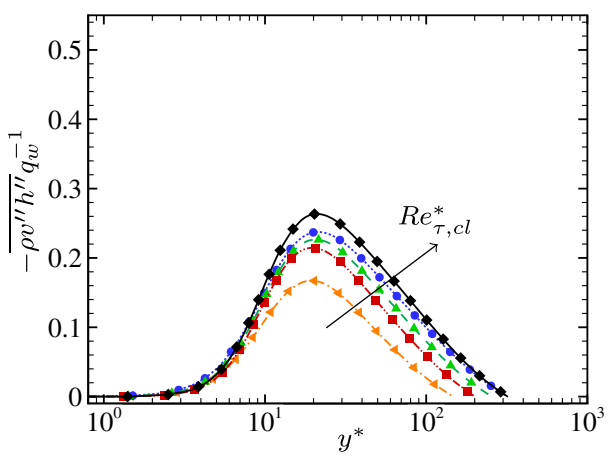

(e)

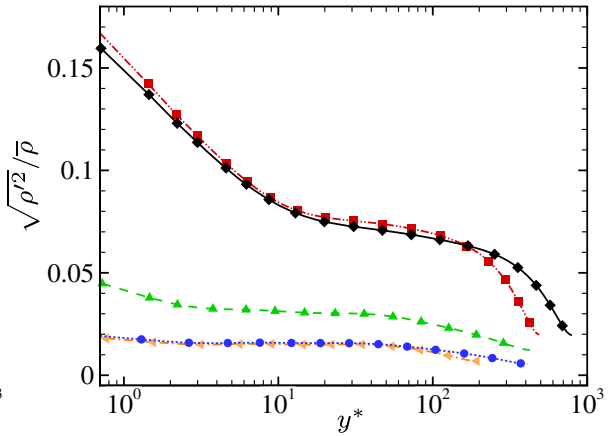

(b)

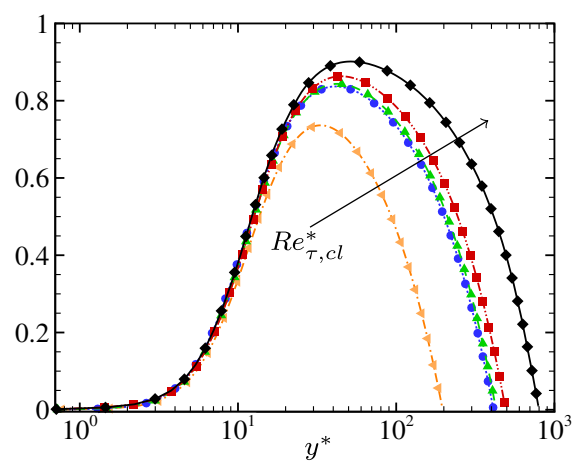

(d)

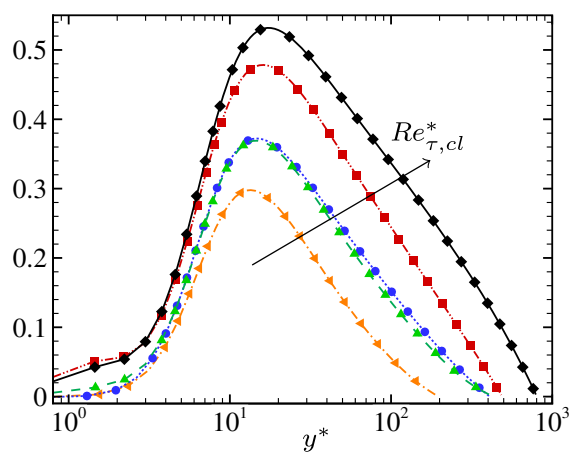

(f)

Fig. 6 a, b Normalized density fluctuations $\left(\sqrt{\rho^{\prime 2}} / \bar{\rho}\right), \mathbf{c}, \mathbf{d}$ Reynolds shear stresses $\left(\overline{\rho u^{\prime \prime} v^{\prime \prime}}{ }^{+}\right)$, and e, $\mathbf{f}$ profiles of $\overline{\rho v^{\prime \prime} h^{\prime \prime}}$ normalized with $q_{w}$ as a function of $y^{*}$ for DNS of air (left column) and PP11 (right column) TCF at various $M_{B}$ and $R e_{B}$. Line legend as in Table 3

\section{A Priori Tests of Turbulence Models}

\subsection{Eddy viscosity models}

DNS data are used to investigate the validity of some popular models for the RANS equations. More specifically, we focus hereafter on the eddy viscosity and turbulent Prandtl 

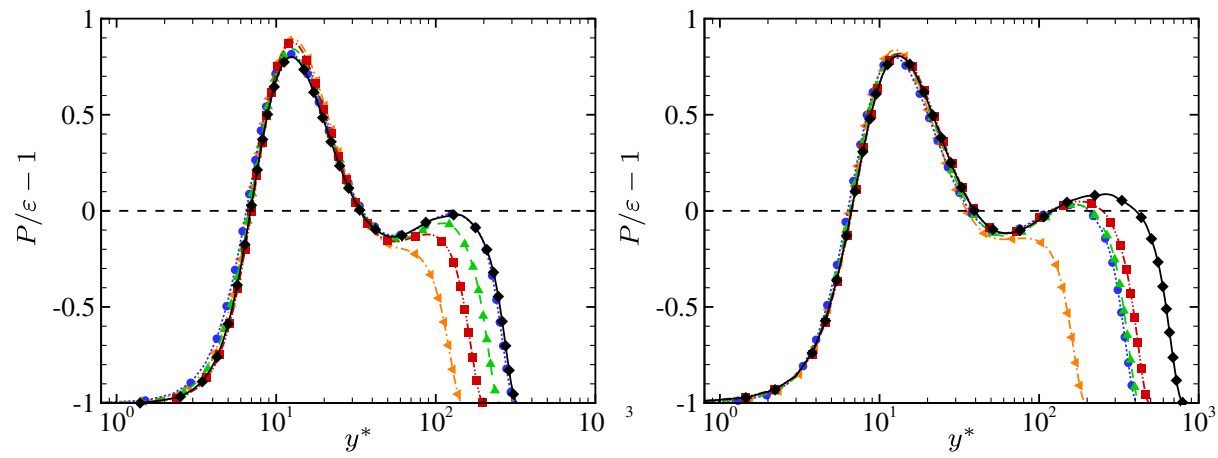

Fig. 7 Production-to-dissipation ratio of turbulent kinetic energy $(P / \varepsilon-1)$ as a function of $y^{*}$ for DNS of air (left column) and PP11 (right column) TCF at various $M_{B}$ and $R e_{B}$. Line legend as in Table 3

number assumptions, commonly used to approximate, respectively, the Reynolds stress tensor and the turbulent heat flux. To this end, the "exact" eddy viscosity and turbulent Prandtl number are computed from DNS data as:

$$
\mu_{t}=\overline{\rho u^{\prime \prime} v^{\prime \prime}}\left(\frac{d \bar{u}}{d y}\right)^{-1} ; \quad P r_{t}=\bar{c}_{p} \mu_{t} / \lambda_{t}
$$

where $\bar{u}$ is the averaged streamwise velocity and $\lambda_{t}=\overline{\rho v^{\prime \prime} h^{\prime \prime}} \bar{c}_{p}\left(\frac{d \bar{h}}{d y}\right)^{-1}$ is a turbulent thermal conductivity, with $\bar{h}$ the averaged static enthalpy. Input quantities required by eddy models are also based on DNS. In this study we restrict our attention to three low-Reynolds variants of the $k-\varepsilon$ model, namely, the Launder-Sharma (LS) [38], the Chien (CH) [39] and the Lam-Bremhorst (LB) [40] models, which assume that the eddy viscosity is proportional to "isotropic" velocity and length scales based on the turbulent kinetic energy $k$ and to the turbulent dissipation $\varepsilon$. These are corrected in the near-wall region to account for turbulence decay in the viscous sublayer and for the wall blocking effect by introducing a damping function $f_{\mu}$ :

$$
\mu_{t}=C_{\mu} f_{\mu} \frac{\rho k^{2}}{\widetilde{\varepsilon}}
$$

with $C_{\mu}$ usually taken equal to 0.09 , and $\varepsilon=\widetilde{\varepsilon}+D$. Using $\widetilde{\varepsilon}$ as the "dissipation variable" instead of $\varepsilon$ was first proposed by Jones and Launder [41] for its computational avantages; $D$ is indeed chosen such that $\widetilde{\varepsilon}=0$ at the wall. However, the definition of $D$ is not the same for all authors. The model-dependent damping functions $f_{\mu}$ and coefficients $D$ for the models retained in this study are given in Table 4, where $R_{t}=\rho k^{2} /(\mu \widetilde{\varepsilon})$ and $R_{y}=\sqrt{k} y / \nu$. Durbin (DB) [15] showed that a more appropriate choice for the velocity scale in the inner

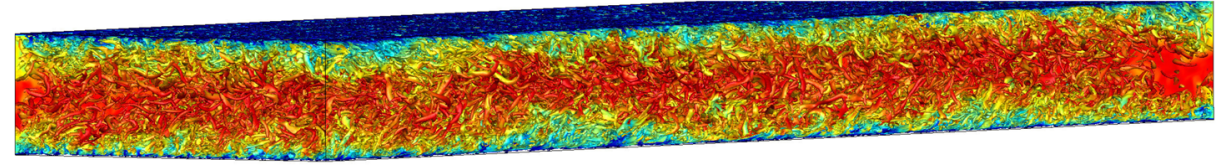

Fig. 8 Isosurface of $Q\left(h / u_{B}\right)^{2}=1$ colored with streamwise velocity for case PM3R7 
Table 4 Damping function $f_{\mu}$ and coefficient $D$ for the $k-\varepsilon$ low- $R e$ turbulence models retained in the study

\begin{tabular}{lll}
\hline Models & $f_{\mu}$ & $D$ \\
\hline (LS) & $\exp \left(\frac{-3.4}{\left(1+R_{t} / 50\right)^{2}}\right)$ & $2 v\left(\frac{\partial \sqrt{k}}{\partial y}\right)^{2}$ \\
(CH) & $1-\exp \left(-0.0115 y^{+}\right)$ & $2 v \frac{k}{y^{2}}$ \\
(LB) & $1-\exp \left(-0.0165 R_{y}\right)\left(1+\frac{20.5}{R_{t}}\right)$ & 0 \\
\hline
\end{tabular}

region is represented by the root-mean square of the wall-normal fluctuating velocity, and reformulated the eddy viscosity as

$$
v_{t}=C \frac{\sqrt{\widetilde{v^{\prime \prime 2}}} k}{\varepsilon}
$$

with $C=0.2$ (the associated line legend is shown in Table 5).

All of the preceding models were initially developed for high Reynolds number and incompressible flows and their behavior is expected to change in presence of compressibility effects, which are different for perfect and dense gases. In particular, at least for perfect gases, friction heating leads to lower effective friction Reynolds numbers than the incompressible case. For this reason, before analyzing the model behavior for compressible flow data, a priori tests of the preceding models are conducted using the DNS database (based on a dealiased semi-spectral method) of Hoyas and Jimenez [16] for incompressible TCF up to $R e_{\tau}=2003$. This provides reference incompressible results and allows to investigate the influence of the flow Reynolds number on model performance. The results are reported in Fig. 9, showing the evolution of eddy viscosity in wall scaling $\left(v_{t}^{+}=v_{t} / v_{w}\right)$ against the inner coordinate $y^{+}$(the associated line legend is shown in Table 5). Most RANS closures for the eddy viscosity fail to match the exact eddy viscosity profile in the near wall region, and may overestimate the eddy viscosity in the outer region. The errors tend to be larger for low-Re flows, characterized by a thicker viscous layer. The LS model exhibits an incorrect trend in the buffer region, where it predicts a locally reversed curvature compared to the DNS data. This local "hump" is more evident for low Reynolds cases (the worst situation being observed for $R e_{\tau_{w}}=180$ where it leads to the appearance of a local extremum) and tends to be damped out at higher $R e_{\tau_{w}}$. This incorrect trend is greatly improved by the $\mathrm{CH}$ and $\mathrm{LB}$ formulation, for which the hump is still present but is only visible for the lowest Reynolds number. For the $\mathrm{CH}$ model, the $D$ term computed from DNS data results in a locally slightly negative modified dissipation near the wall, which is unphysical. For this reason, the tests where conducted by setting $D=0$ for this model, which allows at least to assess the damping function $f_{\mu}$. Among the models using the standard isotropic formulation of $v_{t}, \mathrm{CH}$ provides the most accurate results in the viscous sublayer, where it follows rather closely the DNS curve. The LS, CH and LB models tend to the same solution in the outer region, at least for sufficiently high Reynolds numbers, since the damping functions tend to vanish in this region. Durbin's model provides a much more accurate representation of the $v_{t}$ distribution for all cases, although the eddy viscosity in the outer region is underestimated at low $R e_{\tau}$ and tends to be overestimated more and more as $R e_{\tau}$ increases. On the

Table 5 Turbulent viscosity models and associated line legend

\begin{tabular}{llllll}
\hline Models & Exact & Launder-Sharma & Chien & Lam-Bremhorst & Durbin \\
Line & & nu, & & \\
\hline
\end{tabular}




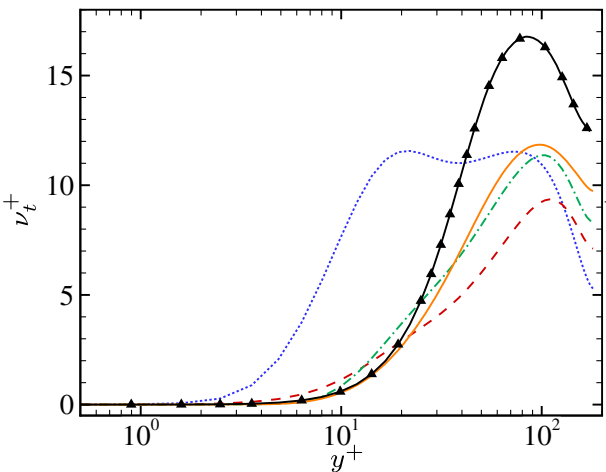

(a) $R e_{\tau_{w}}=180$

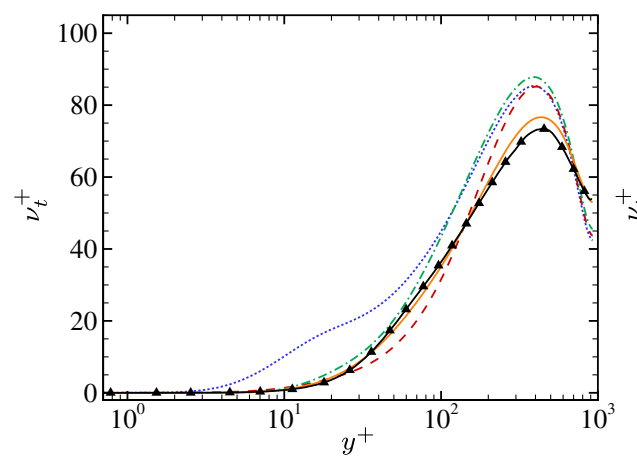

(c) $R e_{\tau_{w}}=950$

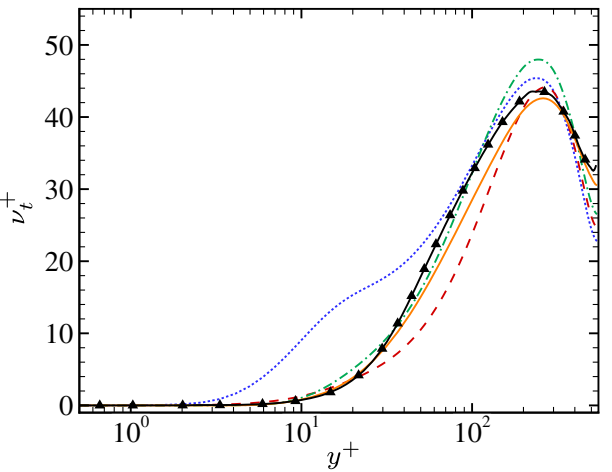

(b) $R e_{\tau_{w}}=550$

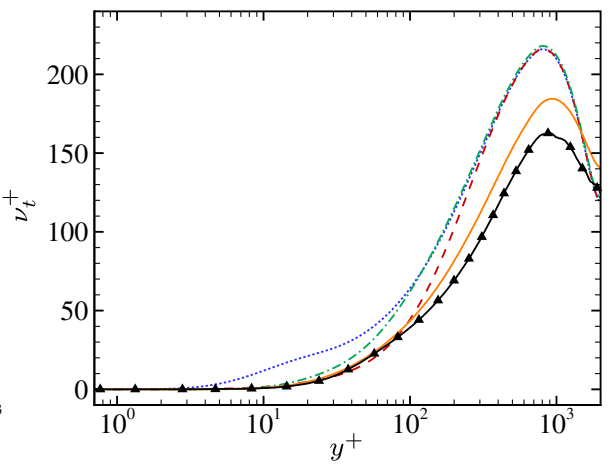

(d) $R e_{\tau_{w}}=2000$

Fig. 9 Normalized turbulent viscosity computed from the DNS databases of [16]. Line legend as in Table 5

other hand, such a trend is also observed for the other models under investigation. In Fig. 10 we report the profiles of $v_{t}^{+}$at selected flow conditions, based on the present compressible DNS both for air and PP11. These correspond to the lowest, intermediate and highest value of $R e_{\tau, c l}^{*}$, respectively. The results are reported as functions of the semi-local coordinate $y^{*}$. It is interesting to observe that, once the semi-local scaling is applied, results for the present compressible cases appear to be rather similar to the incompressible ones, provided that the comparisons are made for similar values of $R e_{\tau, c l}^{*}$. For both air and PP11 flows, the LS model badly overestimates the turbulent viscosity in the viscous sublayer. Chien's model captures better the trend of $v_{t}$ but also overestimates the turbulent viscosity in the outer region for PP11 at the highest Reynolds conditions. Durbin's model provides a reasonably accurate approximation of the $v_{t}$ profile up to part of the logarithmic region. For PP11, the model remains in rather good agreement with DNS up to $y^{*} \approx 150$ and strongly overestimates $v_{t}$ for higher values of $y^{*}$, despite $R e_{\tau, c l}^{*}=800$ for this case, a value for which the models perform rather well when using incompressible DNS data. This discrepancy is probably due to the fact that $\widetilde{v^{\prime \prime}}$, used to form the velocity scale in Durbin's model, tends to take larger values in PP11 due to the liquid-like behavior of the flow properties. The model reacts to such an increase, leading to the observed trend.

Figure 11 shows the eddy viscosity profiles in outer scaling for the highest $R e_{\tau, c l}^{*}$ cases, both for air and PP11. In this scaling, all models except LS capture reasonably well the overall trend. The relative error is roughly of the same order $(\sim 20 \%)$ in all cases. Durbin's 


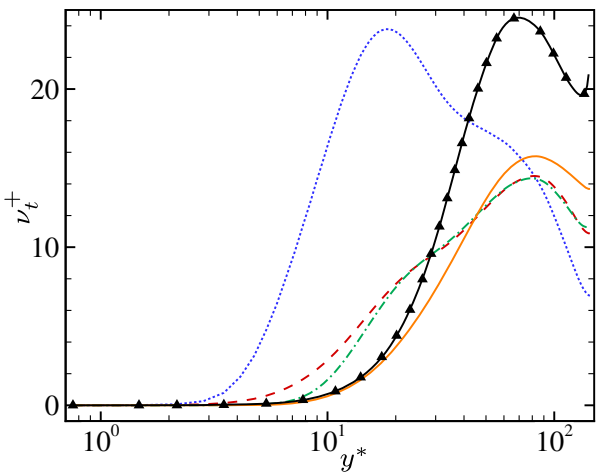

(a) $\operatorname{AM1R3}\left(R e_{\tau}^{*}=147.1\right)$

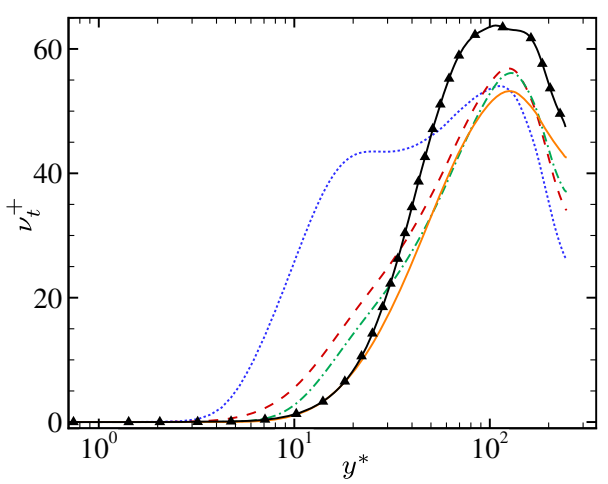

(c) AM2R7 $\left(R e_{\tau}^{*}=252.0\right)$

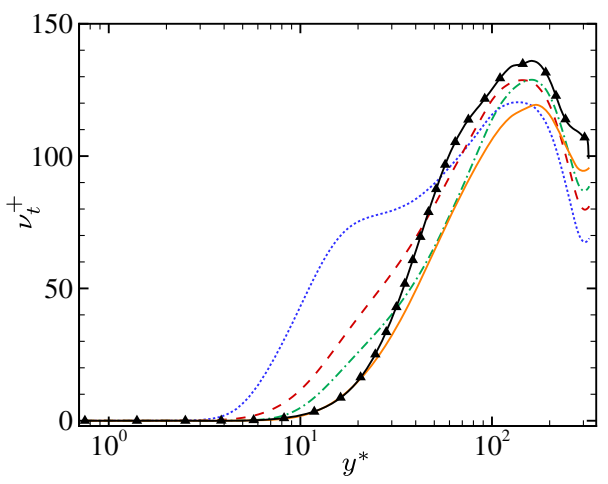

(e)AM3R12 $\left(R e_{\tau}^{*}=324.7\right)$

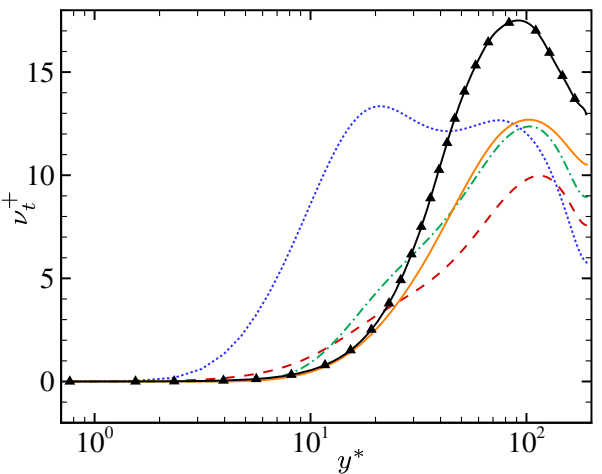

(b) PM1R3 $\left(R e_{\tau}^{*}=196.4\right)$

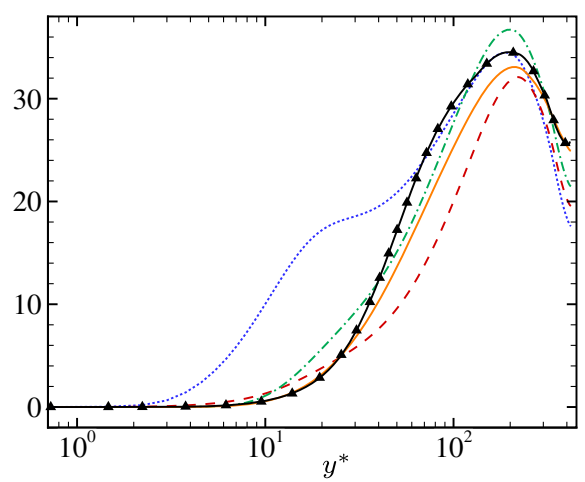

(d) PM2R7 $\left(R e_{\tau}^{*}=428.0\right)$

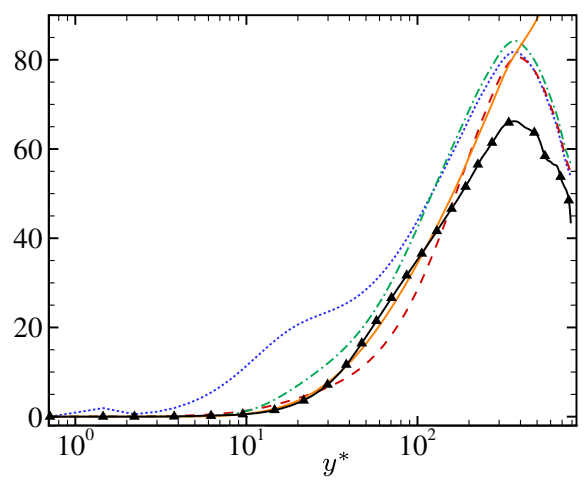

(f) PM3R12 $\left(R e_{\tau}^{*}=800.2\right)$

Fig. 10 Normalized turbulent viscosity for selected flow cases as a function of $y^{*}$. Left column: air; right column: PP11. Line legend as in Table 5

model over-reacts to the increase of $\widetilde{v^{\prime \prime}}$ in the outer region for the dense gas case, leading to a severe overestimation of $v_{t}$ (about $40 \%$ ). The $\mathrm{CH}$ model represents a good compromise in terms of accuracy both for the inner and the outer region. 


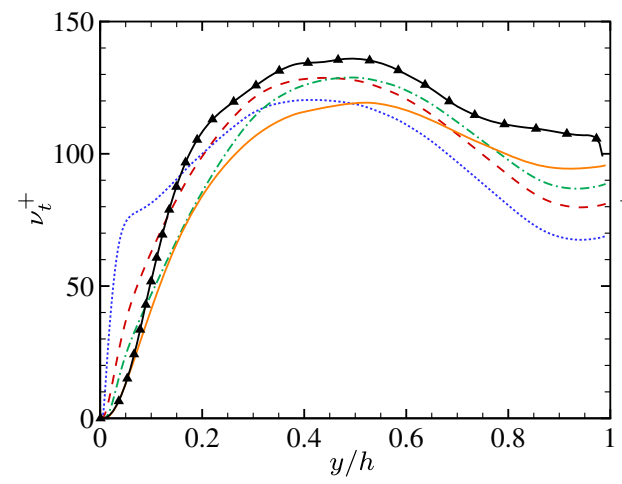

(a) $\operatorname{AM} 3 R 12\left(R e_{\tau}^{*}=324.7\right)$

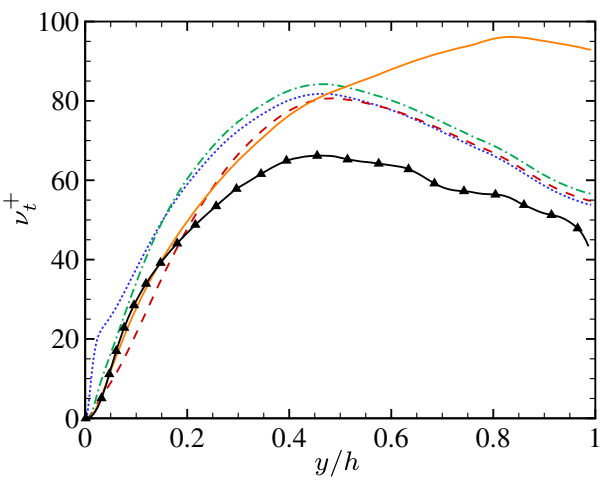

(b) PM3R12 $\left(R e_{\tau}^{*}=800.2\right)$

Fig. 11 Normalized turbulent viscosity for cases AM3R12 (left) and PM3R12 (right) in outer scaling. Line legend as in Table 5

\subsection{Turbulent Prandtl number models}

In Fig. 12 we report the exact turbulent Prandtl number at various $M_{B}$ and $R e_{B}$. For air, $P r_{t}$ follows the trends observed by other authors in the literature [10] and is not constant throughout the flow, in contrast with the classical assumption $P r_{t} \approx 0.9$. In particular, for the present relatively low-Re flow, $P r_{t}$ exhibits only a small plateau around $y^{*} \approx 100$ where its value is close to the standard "constant" value of 0.9 . In the outer region, $P r_{t}$ decreases with $y^{*}$, while in the inner region it exhibits a local maximum at about $y^{*}=50$ and tends to approximately 1.1 at the wall. The local extremum at $y^{*}=50$ tends to decrease as the centerline semi-local friction Reynolds number increases. This is highlighted by the closeup view in Fig. 12c, where results from the high-Reynolds compressible DNS of Modesti and Pirozzoli [32] (for which $R e_{\tau, c l}^{*}=677$ ) is also reported. In the immediate neighborhood of the wall $\left(y^{*} \lesssim 5\right), P r_{t}$ tends to increase, the precise behavior depending on $M_{B}$ and (less markedly) on $\operatorname{Re}_{B}$.

For PP11, the overall behavior is rather close to that of the perfect gas over most of the channel height $\left(y^{*} \gtrsim 5\right)$. Specifically, the 0.9 value remains approximately valid also for a dense gas flow, at least in the outer region. The dense gas solution exhibits even a larger '0.9' plateau than the air flow, located at $y^{*} \approx 120$, most likely because of the higher local $R e_{\tau}^{*}$. The local maximum around $y^{*} \approx 50$ is also observed for the dense gas, although the corresponding $\operatorname{Pr}_{t}$ value appears to be more sensitive to the flow condition than in the case of air. Note that in this region the local molecular Prandtl number is comprised between 2.3 and 2.5 for the different flow cases (to be compared with $\operatorname{Pr}=$ const $=0.7$ for air). A drastically different behavior is observed in the viscous sublayer $\left(y^{*} \lesssim 5\right)$, where the local molecular Prandtl number is much higher (varying approximately from 2.6 for $M_{B}=1.5$ to 3.9 for $M_{B}=3$ ) and the local $R e_{\tau}^{*}$ lower than in air (see Fig. 4). In this region $\left(y^{*} \approx 2\right.$ ), $\mathrm{Pr}_{t}$ exhibit another local maximum (more or less pronounced according to the flow conditions) and tends to values lower than 1 at the wall $(\approx 0.4$ at the highest Mach number). Inspection of the DNS data for $v_{t}$ and $\lambda_{t}$, reported in Figs. 13 and 14, suggests the following explanation: for air, both $v_{t}$ and $\lambda_{t}$ decrease approximately at the same rate $\left(O\left(y^{* p}\right)\right)$ when $y^{*} \rightarrow 0$. The exponent $p \approx 3.1 \div 3.9$ is higher than the incompressible value due to friction heating effects that tend to thicken the viscous sublayer. Since $c_{p}=$ const for air, 


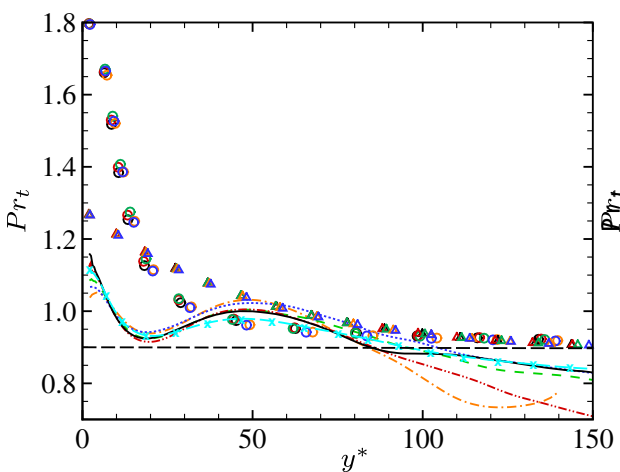

(a) Air

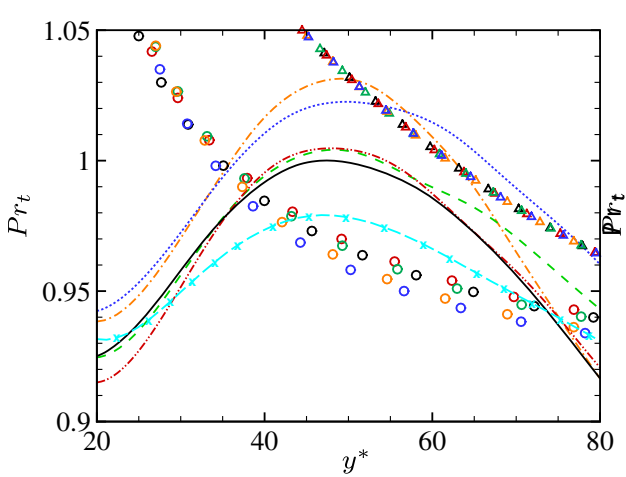

(c) Air, closeup view for $20 \leqslant y^{*} \leqslant 80$

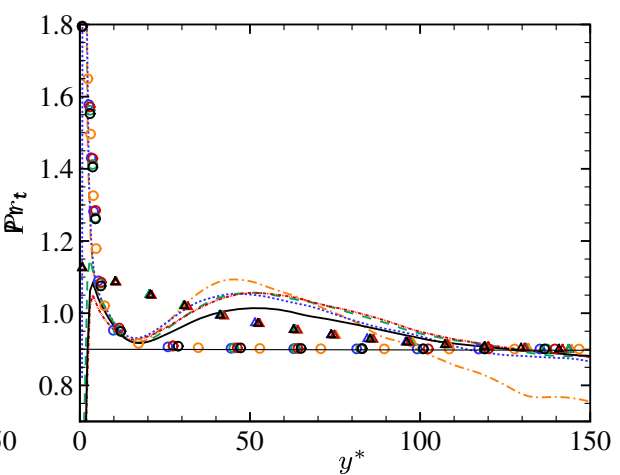

(b) PP11

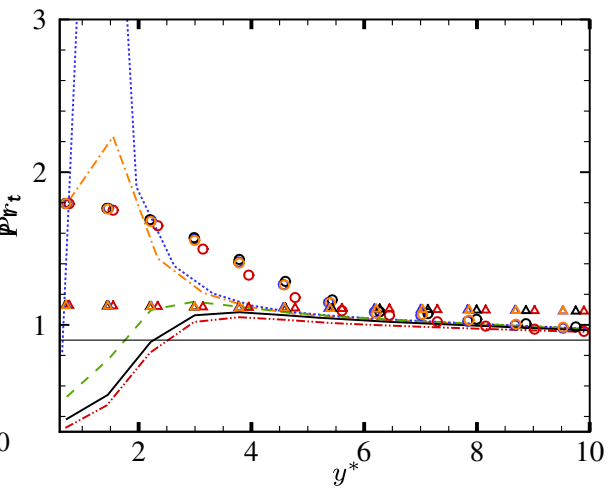

(d) PP11, closeup view for $y^{*} \leqslant 10$

Fig. 12 Exact turbulent Prandtl number $P r_{t}$ for air (top left) and PP11 (toright), with close-up view in the region $20<y^{*}<80$ for air (bottom left) and in the near-wall region $\left(y^{*}<15\right.$ ) for PP11 (bottom right) at various $M_{B}$ and $R_{B}$. Legend as in Table 3, but without symbols for the sake of clarity. Instead, symbols are for a-priori tests of the models: $\mathbf{\Delta}$ : Cebeci model, $\mathbf{O}$ : Kays and Crawford model. Symbol colors denote the case, in accordance with line colors of Table 3. For air, cyan dashed line ( - - X- - ') corresponds to data from [32]. Horizontal line is set at $\operatorname{Pr}_{t}=0.9$

then $\mathrm{Pr}_{t}$ tends toward a constant nonzero value. For PP11, $\lambda_{t}$ decreases approximately at the same rate as $v_{t}$ up to the verge of the thermal viscous sublayer. Once again the exponents differ from the classical value for incompressible constant property flows due to flow property variations in the present compressible DNS. In the dense gas, the mean molecular viscosity behaves the opposite than in perfect gas, and thus the exponent is slightly lower than 3 for PP11. In the thermal viscous sublayer the mean enthalpy gradient changes, leading to a different slope of the turbulent conductivity curve in the near wall region $\left(y^{*} \lesssim 2\right)$. The near-wall slope depends on the Mach and, to a much lesser extent, on the Reynolds number. For $M_{B}=1.5,2.25$ and 3 the near-wall slope is approximately equal to 1.1, 1.8 and 2.9, respectively, with small variations for different Reynolds numbers. For the two highest Mach numbers, the near wall slope for $\lambda_{t}$ is smaller than the one for $v_{t}$, so that the ratio of turbulent viscosity to turbulent conductivity tends to vanish at the wall. However, $\bar{c}_{p}$ increases abruptly when $y^{*} \rightarrow 0$ (following an approximately exponential trend). From the definition of $P r_{t}$ it follows that a local maximum is to be expected in the viscous sublayer. A much more pronounced maximum is found for $M_{B}=1.5$, for which $\bar{c}_{p}$ variations 

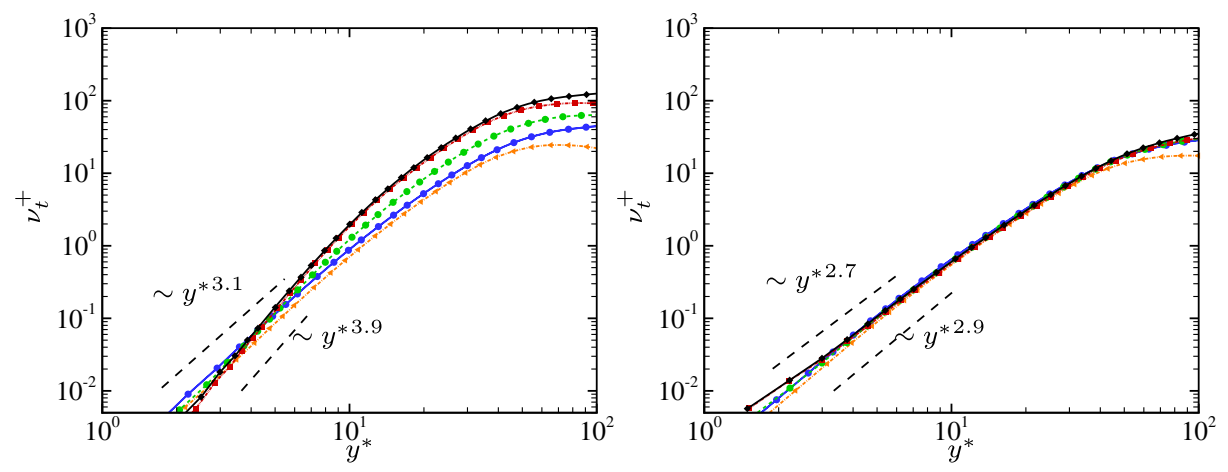

Fig. 13 Trends of the exact eddy viscosity for air (left) and PP11 (right). Legend as in Table 3

are smaller, but $\lambda_{t}$ varies approximately at the same rate as $v_{t}$. Simulations with a finer resolution of the thermal viscous sublayer are planned in the future to further explore the near wall behavior of dense gas cases.

In the same figures we also report for comparison the results of two variable turbulent Prandtl number models. The first one is an analytical model proposed by Cebeci [42], based on van Driest's mixing length model for the eddy viscosity and a similar model for the thermal mixing length:

$$
P r_{t}=\frac{\kappa}{\kappa_{\theta}}\left(\frac{1-\exp \left(-y^{+} / A^{+}\right)}{1-\exp \left(-y^{+} / B^{+}\right)}\right)
$$

with $\kappa=0.41$ the von Karman constant, $\kappa_{\theta}=0.46$ the thermal von Karman constant and $A^{+}$and $B^{+}$Reynolds-number dependent constants. Here we use the variant of Cebeci's model proposed by $\mathrm{Na}$ and Habib [43], also considered by [20, 44], where $A^{+}=26$ and $B^{+}$is expressed as a function of the molecular Prandtl number:

$$
B^{+}=\sum_{i=1}^{5} C_{i}\left(\log _{10} \operatorname{Pr}\right)^{i-1}
$$
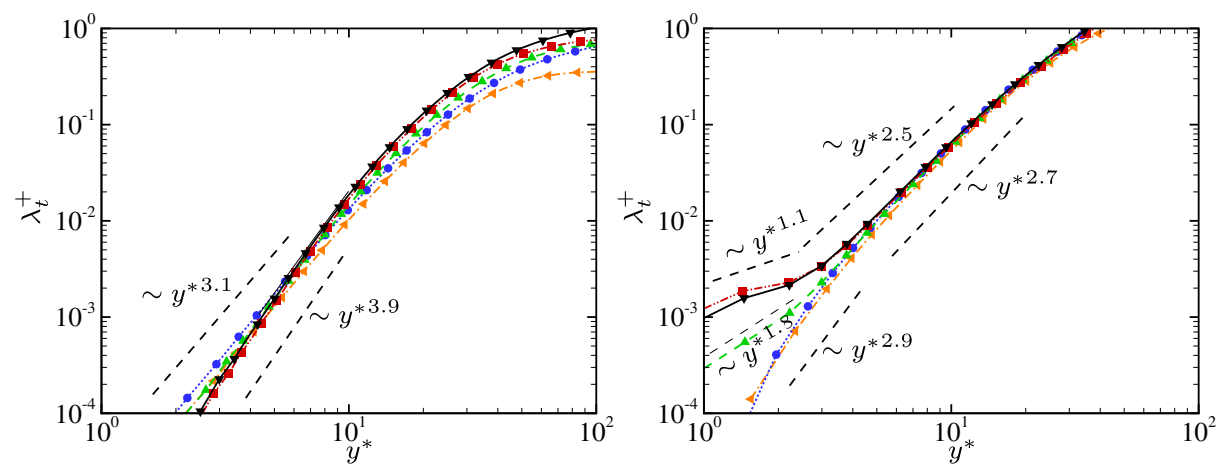

Fig. 14 Trends of the exact non dimensional turbulent thermal conductivity $\lambda_{t}^{+}=\lambda_{t} / \lambda_{w}$ for air (left) and PP11 (right). Legend as in Table 3 
The second model is the empirical model developed by Kays and Crawford [45] for air, with constants rewritten for a generic molecular Prandtl number:

$$
P r_{t}=\left[\frac{1}{2 P r_{t, \infty}}+\frac{0.3 P e_{t}}{\sqrt{P r_{t, \infty}}}-\left(0.3 P e_{t}\right)^{2}\left(1-\exp \left(\frac{-1}{0.3 P e_{t} \sqrt{P r_{t, \infty}}}\right)\right)\right]^{-1}
$$

where $P e_{t}=\operatorname{Pr} v_{t} / v$ is a turbulent Peclet number and $P r_{t, \infty}=0.9$ is the bulk Prandtl number. In both cases, we plot the models as functions of $y^{*}$ to enable comparisons with our compressible DNS and, for PP11, we use a variable molecular Prandtl number, corresponding to the averaged Prandtl number $\overline{P r}$ from the DNS. Both the theoretical and the empirical model predict a maximum of $P r_{t}$ at the wall and a monotonic decrease toward the centerline, where the bulk value is reached. None of them predicts the local maximum in the logarithmic region, which is most probably a low Reynolds number effect, as argued by [46]. The models tend also to overpredict the wall value of $P r_{t}$. None of the models captures the non-monotonic behavior of $P r_{t}$ observed in the thermal viscous sublayer for PP11.

\section{Conclusions}

A priori tests of the validity of some widely-used modeling assumptions for the eddy viscosity and turbulent Prandtl number were carried out for compressible turbulent channel flows of a dense gas, based on direct numerical simulation data. The data have been generated for various bulk Mach and Reynolds numbers and the results were systematically compared to those obtained for air at the same conditions. For a dense gas, the classical $y^{+}$scaling based on the friction velocity fails to collapse thermodynamic profiles and Reynolds stresses at high $M_{B}$, and the semi-local scaling, which accounts for variations of the flow properties, has to be adopted instead. The coupling between dynamic and thermal effects is very small for dense fluid characterized by high specific heats, contrary to air which undergoes significant friction heating (typical of highly compressible flows). Turbulence structure is shown to be little affected by dense gas effects. For the adopted thermodynamic conditions, transport properties exhibit a liquid-like behavior and the local Reynolds number in the outer region is found to be much higher than in corresponding air flows. A priori analyzes for various eddy viscosity models, and more precisely for four low-Reynolds variants of the $k-\varepsilon$ model showed that, except for the Launder-Sharma model which predicts incorrect trends of $v_{t}$ in the near wall region, all of the turbulence models for eddy viscosity are in reasonable qualitative agreement with the DNS data. The agreement with DNS is better as the flow effective Reynolds number increases. In this respect, the modeled eddy viscosity for the dense gas follows more closely the exact one than in perfect gas (at the present high Mach numbers), due to the higher local Reynolds number. However, the eddy viscosity in the outer region is overestimated. An adjustment of the model constants could help improving the results.

Computations of the exact turbulent Prandtl number from the dense gas DNS data highlighted a peculiar behavior of this parameter close to the wall, which peaks more or less abruptely in the thermal viscous sublayer (according to the flow conditions and namely the flow Mach number), instead of tending to a constant near-wall value as in air. This phenomenon, most likely due to the abrupt increase of the fluid specific heat near to the wall, is not captured by the existing turbulent Prandtl models developed for standard gases. The development of more suitable models for $\operatorname{Pr}_{t}$ in dense gases will be the subject of future research. 
Acknowledgements This work was granted access to the HPC resources of GENCI (Grand Equipement National de Calcul Intensif) under the allocation 7332. The authors are grateful to Dr. Davide Modesti for providing the turbulent Prandtl number profile at $R e_{\tau, c l}^{*}=677$. The authors declare that they have no conflict of interest.

\section{References}

1. Kirillov, N.: Analysis of modern natural gas liquefaction technologies. Chem. Pet. Eng. 40(7-8), 401406 (2004)

2. Zamfirescu, C., Dincer, I.: Performance investigation of high-temperature heat pumps with various BZT working fluids. Thermochim. Acta 488, 66-77 (2009)

3. Brown, B., Argrow, B.: Application of Bethe-Zel'dovich-Thompson fluids in organic Rankine cycle engines. J. Propuls. Power 16(6), 1118-1124 (2000)

4. Congedo, P., Corre, C., Cinnella, P.: Numerical investigation of dense-gas effects in turbomachinery. Comput. Fluids 49(1), 290-301 (2011)

5. Thompson, P.: A fundamental derivative in gasdynamics. Phys. Fluids 14(9), 1843-1849 (1971)

6. Cinnella, P., Congedo, P.: Inviscid and viscous aerodynamics of dense gases. J. Fluid Mech. 580, 179217 (2007)

7. Sciacovelli, L., Cinnella, P., Content, C., Grasso, F.: Dense gas effects in inviscid homogeneous isotropic turbulence. J. Fluid Mech. 800(1), 140-179 (2016)

8. Sciacovelli, L., Cinnella, P., Grasso, F.: Small-scale dynamics of dense gas compressible homogeneous isotropic turbulence. J. Fluid Mech. 825, 515-549 (2017)

9. Sciacovelli, L., Cinnella, P., Gloerfelt, X.: Direct numerical simulations of supersonic turbulent channel flows of dense gases. J. Fluid Mech. 821, 153-199 (2017)

10. Huang, P., Coleman, G., Bradshaw, P.: Compressible turbulent channel flows: DNS results and modelling. J. Fluid Mech. 305, 185-218 (1995)

11. Patel, V., Rodi, W., Scheuerer, G.: Turbulence models for near-wall and low reynolds number flows: A review. AIAA J. 23(9), 1308-1319 (1985)

12. Launder, B.: Second moment closure: Methodology and practice. Tech. rep., Proceedings of the Ecole d'Eté d'Analyse Numérique-Modélisation Numérique de la Turbulence. Clamart, France (1982)

13. Shih, T.: An improved k-epsilon model for near-wall turbulence and comparison with direct numerical simulation. Tech. rep., NASA TM 103221 (1990)

14. Kim, J., Moin, P., Moser, R.: Turbulence statistics in fully developed channel flow at low reynolds number. J. Fluid Mech. 177, 133-166 (1987)

15. Durbin, P.A.: Near-wall turbulence closure modeling without "damping functions". Theor. Comput. Fluid Dyn. 3(1), 1-13 (1991)

16. Hoyas, S., Jiménez, J.: Scaling of the velocity fluctuations in turbulent channels up to $R e_{\tau=2003}$. Phys. Fluids 18(011), 702 (2006)

17. Karimpour, F., Venayagamoorthy, S.: Some insights for the prediction of near-wall turbulence. J. Fluid Mech. 723, 126-139 (2013)

18. He, S., Kim, W., Bae, J.: Assessment of performance of turbulence models in predicting supercritical pressure heat transfer in a vertical tube. Int. J. Heat Mass Transf. 51, 4659-4675 (2008)

19. Pecnik, R., Patel, A.: Scaling and modelling of turbulence in variable property channel flows. J. Fluid Mech. 823, R1-1-11 (2017)

20. Irrenfried, C., Steiner, H.: DNS based analytical P-function model for RANS with heat transfer at high prandtl numbers. Int. J. Heat Mass Transf. 66, 217-225 (2017)

21. Gerolymos, G., Vallet, I.: Pressure, density, temperature and entropy fluctuations in compressible turbulent plane channel flow. J. Fluid Mech. 757, 701-746 (2014)

22. Martin, J., Hou, Y.: Development of an equation of state for gases. AIChE J. 1(2), 142-151 (1955)

23. Chung, T., Ajlan, M., Lee, L., Starling, K.: Generalized multiparameter correlation for nonpolar and polar fluid transport properties. Ind. Eng. Chem. Res. 27(4), 671-679 (1988)

24. Cramer, M., Park, S.: On the suppression of shock-induced separation in bethe-zel'dovich-thompson fluids. J. Fluid Mech. 393, 1-21 (1999) 
25. Cramer, M., Tarkenton, G.: Transonic flows of Bethe-Zel'dovich-Thompson fluids. J. Fluid Mech. 240, 197-228 (1992)

26. Poling, B., Prausnitz, J., O'Connell, J., Reid, R.: The properties of gases and liquids, vol. 5. McGrawHill, New York (2001)

27. Cramer, M.: Negative nonlinearity in selected fluorocarbons. Phys. Fluids A 1(11), 1894-1897 (1989)

28. Gloerfelt, X., Berland, J.: Turbulent boundary-layer noise: direct radiation at mach number 0.5. J. Fluid Mech. 723, 318-351 (2013)

29. Bogey, C., Bailly, C.: A family of low dispersive and low dissipative explicit schemes for flow and noise computations. J. Comput. Phys. 194(1), 194-214 (2004)

30. Bogey, C., De Cacqueray, N., Bailly, C.: A shock-capturing methodology based on adaptative spatial filtering for high-order non-linear computations. J. Comput. Phys. 228(5), 1447-1465 (2009)

31. Foysi, H., Sarkar, S., Friedrich, R.: Compressibility effects and turbulence scalings in supersonic channel flow. J. Fluid Mech. 509, 207-216 (2004)

32. Modesti, D., Pirozzoli, S.: Reynolds and mach number effects in compressible turbulent channel flow. Int. J. Heat Fluid Flow 59, 33-49 (2016)

33. Morinishi, Y., Tamano, S., Nakabayashi, K.: Direct numerical simulation of compressible turbulent channel flow between adiabatic and isothermal walls. J. Fluid Mech. 502, 273-308 (2004)

34. Zonta, F., Marchioli, C., Soldati, A.: Modulation of turbulence in forced convection by temperaturedependent viscosity. J. Fluid Mech. 697, 150-174 (2012)

35. Lee, J., Jung, S., Sung, H., Zaki, T.: Effect of wall heating on turbulent boundary layers with temperaturedependent viscosity. J. Fluid Mech. 726, 196-225 (2013)

36. Patel, A., Peeters, J., Boersma, B., Pecnik, R.: Semi-local scaling and turbulence modulation in variable property turbulent channel flows. Phys. Fluids 27(9), 095,101 (2015)

37. Trettel, A., Larsson, J.: Mean velocity scaling for compressible wall turbulence with heat transfer. Phys. Fluids 28(2), 026,102 (2016)

38. Launder, B., Sharma, B.: Application of the energy-dissipation model of turbulence to the calculation of flow near a spinning disc. Lett. Heat Mass Trans. 1(2), 131-137 (1974)

39. Chien, K.Y.: Predictions of channel and boundary-layer flows with a low-Reynolds-number turbulence model. AIAA J. 20(1), 33-38 (1982)

40. Lam, C., Bremhorst, K.: A modified form of the k- $\varepsilon$ model for predicting wall turbulence. J. Fluids Eng. 103(3), 456-460 (1981)

41. Jones, W., Launder, B.: The prediction of laminarization with a two-equation model of turbulence. Int. J. Heat Mass Transf. 15(2), 301-314 (1972)

42. Cebeci, T.: A model for eddy conductivity and turbulent Prandtl number. J. Heat Transf. 95, 227-234 (1973)

43. Na, T., Habib, I.: Heat transfer in turbulent pipe flow based on a new mixing length model. Appl. Sci. Res. 28, 302-314 (1973)

44. Pirozzoli, S., Bernardini, M., Orlandi, P.: Passive scalars in turbulent channel flow at high Reynolds number. J. Fluid Mech. 788, 614-639 (2016)

45. Kays, W., Crawford, M., Weigand, B.: Convective heat and mass transfer. McGraw-Hill, New York (1980)

46. Kays, W.: Turbulent Prandtl number - where are we ASME J. Heat Transf. 116(2), 284-295 (1994) 